VISCOSITY OF AQUEOUS SOLUTIONS OF SODIUM PALMITATE, ETC. 347

\title{
XLIII.-The Viscosity of Aqueous Solutions of Sodium Palmitate and the Influence of Electrolytes on the Same.
}

By Frederick Denny Farrow.

IT is now well established that in not too dilute aqueous solutions the alkali salts of the higher fatty acids are to be classed as electrolytic colloids, since these solutions conduct the electric current, and yet show relatively small changes of vapour pressure and boiling point. It was of interest, therefore, to examine the viscosity of such solutions and the effect of electrolytes thereon.

This question possesses also some considerable importance from a technical point of view, as will be explained in the discussion of the results obtained.

Materials.-The salt employed for these experiments was sodium palmitate. This was obtained as an anhydrous, white powder, which dissolved in hot water to produce clear solutions. It was found that these solutions were identical in viscosity with those obtained by dissolving pure palmitic acid in the calculated amount of aqueous sodium hydroxide. The simpler procedure was therefore adopted of using the anhydrous solid palmitate.

The water used in making all solutions was the laboratory distilled water. This was boiled to free it from dissolved gases, and was filtered whilst hot through cotton-wool. It was kept when necessary in well-corked, steamed-out, Jena-glass flasks.

The sodium hydroxide solution was prepared from clean metallic sodium and water. Due care was taken in its preparation and storage to exciude carbon dioxide. By titration with $N / 10$-hydrochloric acid the solution was found to contain 0.687 gram-molecule of sodium hydroxide per 100 grams of solution.

The potassium and sodium chlorides were Kahlbaum's purest, and were free from sulphates.

Methods of Measurement and A pparatus.-The viscosity measurements were made by means of an apparatus similar to one devised by Scarpa (Gazzetta, 1910, 40, 271), and using a formula tested by him to calculate viscosity. 
Shortly, the method consists in measuring the time, $t_{1}$, which is occupied in drawing up, through a vertical capillary tube, sufficient liquid to fill a bulb at the top of this tube, and the time, $t_{2}$, which this volume takes in flowing out from the bulb under the weight of the liquid. Given constant conditions, the viscosity of any liquid is proportional to the expression $t_{1} t_{2} / t_{1}+t_{2}$.

The advantage of the method lies in the fact that no density measurements are necessary, and that it is immaterial whether equal volumes of liquid are used in the different experiments. For soap solutions these advantages are very real, as the readiness with which such solutions solidify, and their tendency to form foam,

Fig. 1.

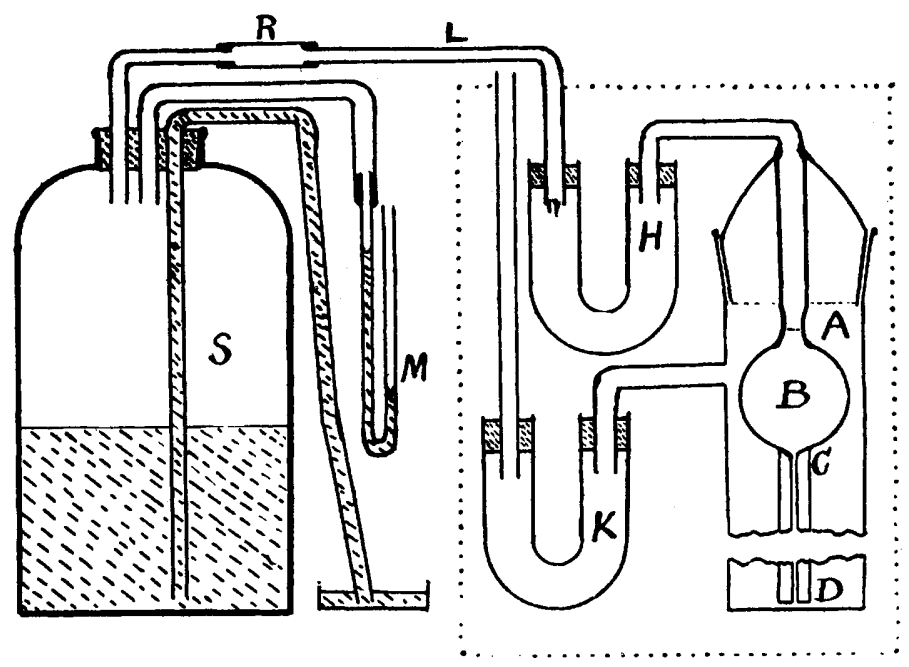

Viscometer and auxiliary apparatus.

makes them exceedingly difficult to measure exactly in pyknometers, or to deliver free from bubbles.

The form of viscometer used was a modification of that used by Scarpa (loc. cit.). The modification consisted in substituting a ground-glass joint for the cork or rubber stopper of Scarpa's apparatus. Fig. 1 is a diagrammatic sketch (not to scale) of the viscometer and accessory apparatus.

$A$ is a glass vessel, with side-tubes as shown, and with a glass tube sealed through its ground-in stopper. This tube is in connexion with the bulb $B$, from the bottom of which leads the capillary tube, $C D$. $H, K$ are guard-tubes containing loosely-packed glass-wool moistened with dilute solution of sodium hydroxide. This packing 
serves to prevent access to the experimental solutions, of carbon dioxide, and of dust, and at the same time checks distillation from, and consequent concentration of, these solutions. $K$ is open to the air on one side, and has a glass tube carried from it to above the water surface of the thermostat, whilst $H$ can be joined, as shown, to the aspirator $S$. The connexion between $R$ (a rubber tube) and $L$ can be undone at will, $R$ being then closed by a spring pinchcock. Suction is applied to $L$ in order to fill the viscometer bulb after the apparatus has been charged with experimental solution.

The working of the aspirator is made sufficiently clear by the diagram. The suction exerted depends on the difference in level between the water in $S$ and that in the outside dish. The measurement of this suction was effected by the water manometer, $M$, which was made to give constantly the same reading. Fine adjustment of the suction was effected by adding to, or withdrawing from, the water in the open dish.

The parts of the apparatus surrounded by a dotted line are enclosed in the thermostat. The viscometer is clamped in a holder designed to secure its accurate vertical adjustment.

Two viscometers were used, of which the essential dimensions were :

$\begin{array}{cc}\text { Viscometer. } & \text { Vol. of bulb. } \\ A \ldots \ldots \ldots . & 8 \cdot 2 \text { c.c. }\end{array}$ Diam. of capillary.
$0.4 \mathrm{~mm}$.
$0.6 \mathrm{~m}$

Length of $C-D$. $10.0 \mathrm{~cm}$. B.......... $4 \cdot 5$, 9.5,

Time.-The times of flow were measured with the aid of a stop. watch, which was kept whilst in use at a constant temperature, and was used under similar conditions as to winding. Tested from time to time against a standard chronometer its rate under these conditions was found to be constant. As the measurements are all referred to a water calibration value, the question of the absolute accuracy of the time measurement does not arise.

Temperature.-For all measurements the viscometer was sunk in a gas-heated water-thermostat with glass sides. The temperature of this was maintained at $70 \pm 0.05^{\circ}$.

\section{Experimental.}

The experimental work divided itself naturally into two parts, namely, (1) measurements with solutions of pure sodium palmitate, and (2) measurements in which the effect of additions to these solutions was tested.

(1) Measurements with Pure Solutions.-The solutions were made up by exactly weighing successively the solid palmitate and the water as they were put into a small flask. The latter was then quickly heated nearly to boiling, corked, and suspended in the thermostat ready for use. 
The viscometer being already clamped with its neck above the water, the required amount of the solution was drawn into a previously warmed pipette, the stopper was lifted, and the liquid delivered into the vessel. The stopper was immediately replaced in its proper position, and as quickly as possible the whole apparatus was lowered until the viscometer and guard-tubes were some 2 inches below the surface of the thermostat water. The holder was re-clamped, due care being always paid to its vertical adjustment.

The exact measurement of the volume of liquid for experiment was not required. Nevertheless, in any series of measurements the volumes used were approximately equal. The water pressure of the aspirator was kept at $20 \mathrm{~cm}$. for viscometer $A$, and at $20 \mathrm{~cm}$., $16 \mathrm{~cm}$., and $15 \mathrm{~cm}$. respectively for the different series with viscometer $B$. In any determination several readings were taken of the times of ascent and of descent. In most cases it was found that the initial readings were high, but that after the complete mixing of the liquid by a filling and emptying of the bulb the times of flow became constant. The initial high viscosity was attributed to unavoidable cooling of solutions in being transferred to the viscometer. It was certainly not attributable to any time-change of the chemical or physical nature of the solutions, as it manifested itself only in the measurements made soon after the immersion of the apparatus, and was in no way dependent on the time which had elapsed since the preparation of the solution. In view of the possibility of a gradual change of the nature of the solutions, tests were made of the viscosity from time to time over a complete day. But in no case was there any certain alteration. The following typical example shows both the initial high viscosity and the subsequent constancy over an extended time.

Viscometer $A$.-Concentration $0 \cdot 131$.

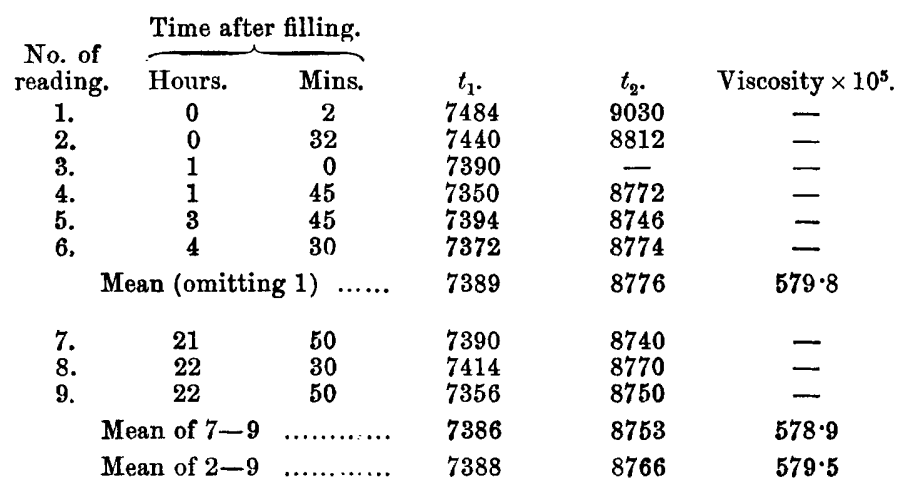


$t_{1}$ and $t_{2}$ respectively are the times (in $1 / 10$ th seconds) of filling and emptying the viscometer bulb. From the times the viscosity $\eta$ is calculated in accordance with the formula:

$$
\eta=\frac{\left(\frac{t_{1} t_{2}}{t_{1}+t_{2}}\right)_{s}}{\left(\frac{t_{1} t_{2}}{t_{1}+t_{2}}\right)_{w}} \cdot \eta_{w},
$$

where $s$ and $w$ indicate solution and water respectively.

Actually the expression $\frac{\eta_{w}}{\left(\frac{t_{1} t_{2}}{t_{1}+t_{2}}\right)_{w}}$ was obtained from measure ments of the times of flow of pure water in the viscometer. The value of $\eta_{w}$ was taken as 0.00407 in absolute units by interpolating from the data of Thorpe and Rodger (Phil. Trans., 1894, 185, A, ii, 397). The calibration data are as follows:

\section{Table I.}

Vol. of Aspirator

Viscometer, water, c.c. gauge, $\mathrm{mm}$. $t_{1}$.

$\begin{array}{rrrrr}A & \ldots \ldots \ldots . & 20 & 200 & 4529 \\ B & \cdots \cdots \cdots & 20 & 200 & 431 \\ B & \ldots \ldots \ldots . & 20 & 160 & 930 \\ B & \cdots \cdots \cdots . . & 10 & 160 & 1173 \\ B & \ldots \ldots \ldots . & 10 & 150 & 1388\end{array}$

$$
V=\frac{\eta_{w}}{\left(\frac{t_{1} t_{2}}{t_{1}+t_{2}}\right)}
$$

$t_{2}$.

7471

$K=\frac{t_{1}+t_{2}}{t_{1} t_{2}} \cdot \quad \begin{gathered}=K \times \\ 0.00407\end{gathered}$

$3546 \quad 1443$

$3345 \quad 1361$

$2033 \quad 8275$

$2033 \quad 8275$

$1911 \quad 7736$

\section{TABLE II.}

\begin{tabular}{|c|c|c|c|c|c|c|}
\hline$c$. & $t_{1}$. & $t_{2}$ & $\frac{t_{1} t_{2}}{t_{1}+t_{2}}$. & $\begin{array}{c}\text { Vis- } \\
\text { conneter. }\end{array}$ & Constant. & $\begin{array}{l}\text { Viscosity } \\
\times 10^{-5} .\end{array}$ \\
\hline 0.026 & 5571 & 6644 & 3026 & A & 1443 & 438 \\
\hline 0.064 & 6224 & 7394 & 3359 & $A$ & 1443 & 485 \\
\hline 0.105 & 6778 & 8023 & 3675 & $A$ & 1443 & 530 \\
\hline $0 \cdot 131$ & 7388 & 8766 & 4006 & $A$ & 1443 & 579 \\
\hline 0.141 & 7638 & 8842 & 4100 & $A$ & 1443 & 592 \\
\hline $0 \cdot 175$ & 8274 & 9780 & 4481 & $A$ & 1443 & 647 \\
\hline 0.187 & 8505 & 10052 & 4605 & $A$ & 1443 & 665 \\
\hline 0.232 & 9579 & 11125 & 5146 & $A$ & 1443 & 743 \\
\hline 0.283 & 10086 & 12062 & 5491 & $A$ & 1443 & 793 \\
\hline 0.287 & 11295 & 12813 & 6002 & $A$ & 1443 & 866 \\
\hline 0.293 & 10787 & 12833 & 5864 & $A$ & 1443 & 847 \\
\hline 0.302 & 11286 & 13470 & 6137 & $A$ & 1443 & 886 \\
\hline 0.329 & 12215 & 14235 & 6575 & $A$ & 1443 & 949 \\
\hline 0.380 & 14400 & 16658 & 7705 & $A$ & 1443 & 1113 \\
\hline 0.451 & 1437 & 3335 & 1002 & $B$ & 1361 & 1363 \\
\hline 0.499 & 1904 & 4319 & 1321 & $B$ & 1361 & 1800 \\
\hline
\end{tabular}

Viscosity of Aqueous Sodium Palmitate Solutions at $70^{\circ}$. 
The composition $(C)$ of table II is expressed as gram-molecules of sodium palmitate per 1000 grams of solution, and the results are shown diagrammatically in Fig. 2.

(2) Experiments with Added Electrolytes.-In these experiments the composition as expressed by the ratio gram-molecules of sodium palmitate/weight total water, was kept constant at the value 0.25 gram-molecule of sodium palmitate in 1000 grams of water; that is, 69.56 grams of sodium palmitate per 1000 grams of water.

The following procedure was adopted in making up the experi-

Fig. 2.

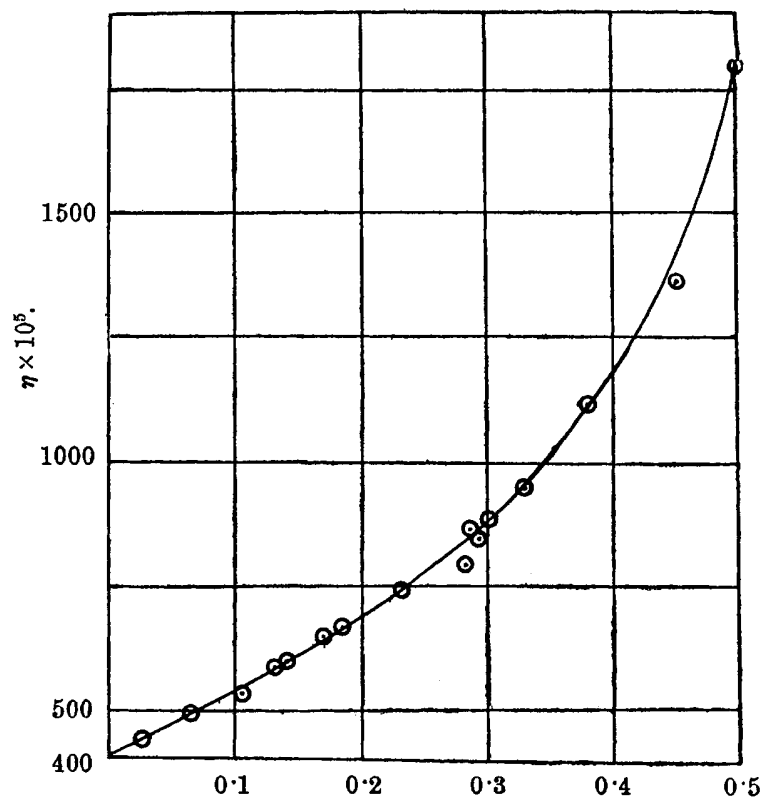

Mols. sodium palmitate per 1000 grams of solution.

mental solutions. Approximately the required amounts of the electrolyte solution and of pure water were weighed separately in a small flask. The total amount of water present was thus obtained, as well as the amount of salt. From the weight of water was calculated the exact amount of palmitate required. This was weighed on a piece of platinum foil, which was dropped into the flask. The latter was then rapidly heated nearly to boiling, corked, and kept in a bath of hot water until solution was accomplished. This never took longer than ten minutes. The measurements were made as with the pure solutions. 


\section{Taber III.}

Mols. of sodium hydroxide per 100 mols. of sodium palmitate.

$$
\begin{array}{r}
0 \cdot 00 \\
0.99 \\
1.61 \\
3 \cdot 18 \\
6 \cdot 28 \\
15 \cdot 05 \\
20 \cdot 18 \\
23 \cdot 05
\end{array}
$$

\begin{tabular}{c|cc} 
& Mols. of sodium hydroxide per \\
$\eta \times 10^{5}$. & 100 mols. of sodium palmitate. & $\eta \times 10^{5}$. \\
733 & $32 \cdot 75$ & 643 \\
723 & $36 \cdot 60$ & 648 \\
706 & $37 \cdot 47$ & 647 \\
705 & $42 \cdot 76$ & 670 \\
681 & $52 \cdot 99$ & 929 \\
630 & $61 \cdot 45$ & 1335 \\
621 & $115 \cdot 32$ & (clear fluid too vis- \\
618 & & cous to flow)
\end{tabular}

TABLE IV.

Mols. of sodium hydroxide (total) per

100 mols. of palmitic acid.

$83 \cdot 4$

$93 \cdot 2$

$\eta \times 10^{5}$.

894

749

TABLE V.

Mols. of sodium chloride per

100 mols. of sodium palmitate. $\eta \times 10^{5}$.

$2 \cdot 01$

$11 \cdot 18$

$16 \cdot 42$

$27 \cdot 97$

$31 \cdot 39$

$61 \cdot 50$

\section{6}

640

625

616

632

1767
Mols. of potassium chloride per 100 mols. of sodium palmitate. $\eta \times 10^{5}$.

$1 \cdot 34$

$12 \cdot 84$

$25 \cdot 60$

$50 \cdot 24$

$64 \cdot 94$

694

622

600

723

1079

Potassium and sodium chlorides. $24 \cdot 6 \mathrm{NaCl}$ $21.9 \mathrm{KCl}$

Total $\overline{46 \cdot 5}$

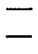

758

\section{Discussion of Results.}

Little success has so far been attained in the treatment from a theoretical point of view of the results of viscosity measurements. More particularly is this the case when dealing with aqueous solutions. With regard to these the attempts which have been made to find formulæ expressing the results of measurements have led to a partial success. The formulæ, however, have been entirely empirical, and as such are of little value in increasing our knowledge of the true meaning of viscosity. No attempt will therefore be made to discuss the concentration-viscosity curve, as we have no knowledge of many of the factors which are necessary for a quantitative investigation of the results obtained.

Turning to the second series of experiments it will be seen that there is an explanation required for the minima which occur on the three curves obtained. The curves for the addition of electrolyte (Fig. 3) resemble the viscosity-concentration curves of certain electrolyte solutions at low temperatures. Such solutions show a 
viscosity minimum at a small concentration of solute. It has been shown, however (Taylor, Proc. Roy. Soc. Edin., 1905, 25, 227), that this initial "negative viscosity" becomes less marked as the temperature is raised, and that it disappears entirely at temperatures above $30^{\circ}$. There can therefore be no question of the minimum observed in the present work being of the same nature as that just mentioned.

When the minimum in the present case had been established for

Fig. 3.

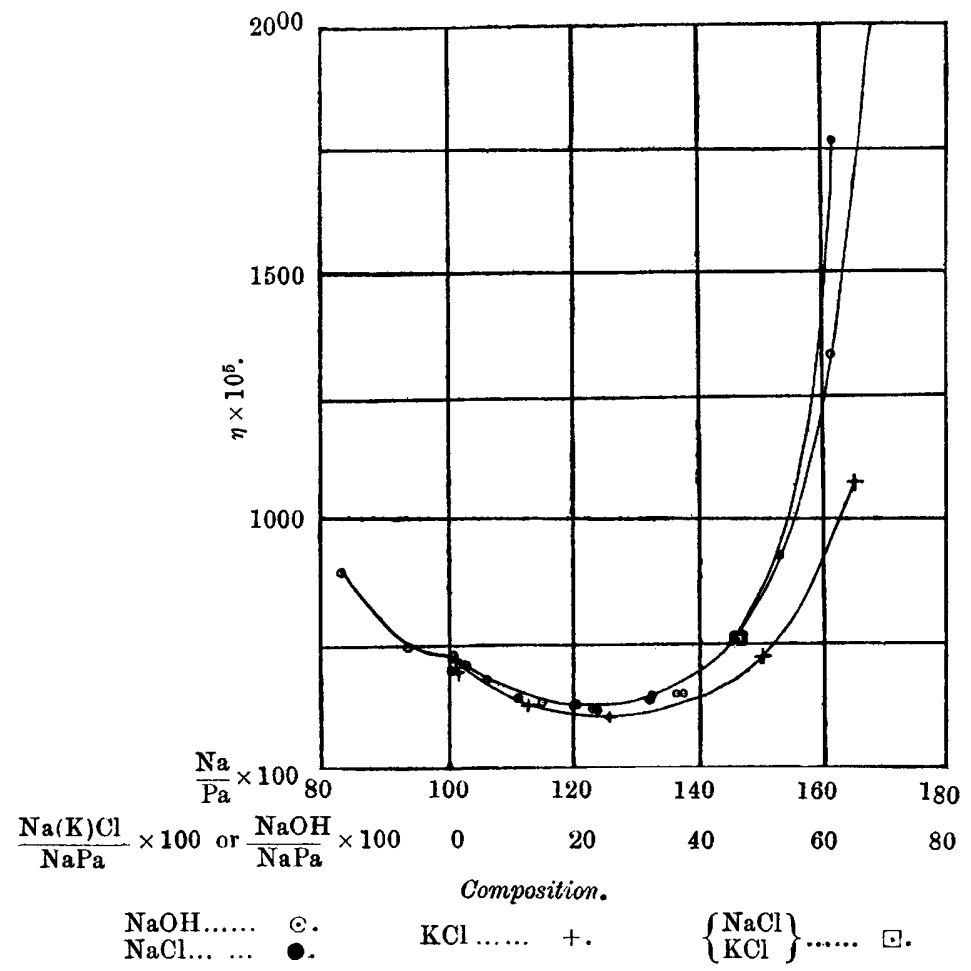

sodium hydroxide alone, it was considered that this could be explained by assuming an initial retardation of the hydrolysis of the sodium palmitate, with a subsequent salting-out action making itself felt on the right-hand branch of the curve. This "chemical" explanation of the initial drop in viscosity had to be abandoned when the practically coincident curve for sodium chloride was obtained. This conclusion is further justified by the precisely similar but quantitatively divergent curve for potassium chloride. 
It seems probable also from other results that the behaviour of soap solutions in the way described is attributable to their functioning as colloids. Woudstra (Zeitsch. Chem. Ind. Kolloide, 1911, 8, 73), working with colloidal ferric hydroxide, has found that, plotting viscosity against concentration of electrolyte, the curve shows an initial fall, with subsequent rapid rise. The smallness of the effect observed in this case is undoubtedly due to the low concentration of the colloidal substance. In the case of gelatin (Gokun, Zeitsch. Chem. Ind. Kolloide, 1908, 3, 84) a similar effect has been observed, although the minimum found for measurements with freshly prepared solutions disappears if the viscosity is determined after a lapse of several hours. The complicating time effect was not observed with the soap solutions, being probably eliminated by the method of their preparation. It appears probable that the minima observed in all three cases are of the same nature, and express a general property of colloidal solutions. That soap solutions judged by the usual criteria are of this nature is well known (see Donnan and White, Trans., 1911, 94, 1668; also footnote to the present paper). They may therefore be regarded in a certain sense as twophase systems, in which the disperse phase is relatively concentrated, that is, contains much higher ratios of alkali and fatty acid to water than the relatively much more dilute and continuous "water phase." A portion of the water in these "colloidal particles" may be, moreover, "bound" or adsorbed. The water phase will contain a relatively small amount of the fatty-acid salt and some alkali hydroxide, the product of the hydrolysis of the soap. If, however, excess of electrolyte is present in the aqueous phase, the activity of the water in this phase is diminished, and there is a tendency for it to decrease in bulk at the expense of the water in systems in contact with it.* In the present case water may be lost by the disperse phase, with a consequent diminution of the bulk of the latter. It is this shrinkage of the colloid particles due to dehydration which is expressed by the left-hand falling branch of the curves.

There is another action of dissolved electrolytes to be considered, namely, that which results in the precipitation of suspended or colloidaliy-dissolved substances. That this action is exerted in soap solutions is shown by the fact that soap is "salted-out" by sodium chloride. It can well be imagined that actual precipitation is preceded by the formation in solution of aggregates; and it is suggested that the presence in the solutions, of such aggregates the

\footnotetext{
* If these systems are bounded by semipermeable membranes, and contain "more active" water, that is, more dilute solutions than the surrounding liquid, the transference is well known as the ordinary phenomenon of osmosis.
} 
size of which increases with the salt concentration, will account for the rapidly rising curve to the right of the minimum. This suggestion assumes that increasing viscosity characterises any decrease of dispersity of a colloidal solution. Woudstra (loc. cit.) has shown this to be the case for several inorganic colloids, and it seems reasonable to suppose that the same action is taking place in the present case. A test of this hypothesis would be to compare the ultramicroscopic appearances of palmitate solutions with different amounts of added electrolytes. This has not so far been found possible owing to experimental difficulties. It is hoped, however, soon to proceed with investigations on these lines.

Application to Technical Practice.-Consider the curves of Fig. 4. Although the present work has determined only one such curve, it seems hardly open to doubt that for different concentrations of soap

FIG. 4.

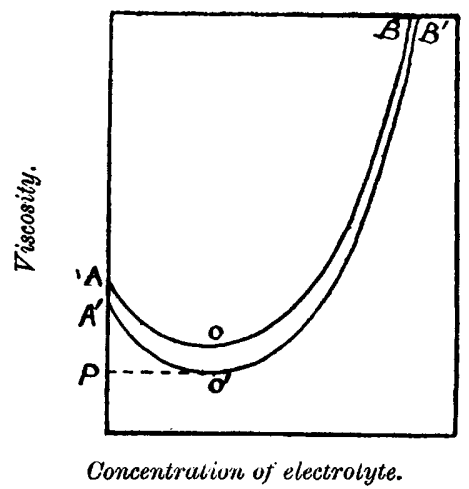

curves would be obtained approximately parallel to this. Let $A O B$ and $A^{\prime} O^{\prime} B^{\prime}$ be two such curves for the soap concentrations $x$ and $y$. If now we start from $O$, the point of maximum fluidity on the upper curve, and wish to reduce the soap concentration to $y$, this can be done by adding the required amount of pure water. In that case the electrolyte present is also diluted, and therefore the viscosity is brought to a point on $A^{\prime} O^{\prime}$ some distance to the left of $O^{\prime}$. We may even by a proper relation between the original concentration and the dilution required arrive at a point above $O$, the dilute solution being thus more viscous than the original. If we add not pure water, but salt solution, it will be possible so to choose the strength of this that in obtaining the soap-concentration $y$ the electrolyte concentration is made equal to $O^{\prime} P$. In this way the solution will have been diluted in the most efficient way possible if fluidity is desired. An over-concentrated salt solution will, of 
course, lead to points on $O^{\prime} B^{\prime}$ representing rapidly increasing viscosity the more concentrated the solution added.

A case apparently analogous to that just dealt with occurs at a certain stage in the clear boiling of soap. It may become necessary to dilute the mass, which consists of sodium salts of mixed fatty acids, a certain amount of free sodium hydroxide, as well as smaller quantities of chloride and carbonate. To effect the dilution it is found necessary to add a solution of common salt of a definite concentration, which depends on the nature of the mixture present. Pure water will not mix readily with the material, whilst too concentrated brine precipitates or "cuts" the soap. Although an analogy between the two cases is obvious, it is not suggested that the technical problem can be adequately dealt with by the simple theory sketched above. The soap-boiler has complex mixtures of composition varying from day to day. He relies at present on empirical tests, the result of the tradition of the trial and error of generations. It may be, however, that viscosity measurements would serve as a further guide as to the condition of the materials in the various stages of soap manufacture.*

In conclusion, I wish to thank Professor Donnan, who suggested this work, for valuable advice, and for the interest which he has shown throughout its course.

The Muspratt Laboratory of Physical and Electro-Chemistry, UNIVERSITY OF LIVERPOOL.

\footnotetext{
* When the experimental work of this paper was complete, my attention was directed to a paper by Mayer, Schaefer, and Terroine (Compt. rend., 1908, 146, 484). These authors deal with various chemical and physical properties of the sodium salts of a series of fatty acids. They state that the viscosity of soap solutions passes through a minimum in passing from acid to alkaline solution, and that the minimum lies on the alkaline side of the neutral point. The facts that no exact data of any sort are given, and that no fuller publication appears to have taken place, are held to justify the present paper. Moreover, the extension of the work to salts other than sodium hydroxide increases its scope in an important manner.
} 